Clinical factors were not associated with $\mathrm{r}_{c} \mathrm{SO}_{2}$ and FTOE, but $\mathrm{r}_{\mathrm{c}} \mathrm{SO}_{2}$ was nearly significantly positively associated with midazolam at day $2(\mathrm{p}=0.05)$, and negatively with $\mathrm{pCO}_{2}$ at day $3(\mathrm{p}=0.051)$.

Conclusions Highest $\mathrm{R}_{\mathrm{c}} \mathrm{SO}_{2}$-values and lowest FTOE-values were seen on day two, suggesting decreased oxygen consumption, possibly as a consequence of midazolam treatment. Even so, treatment with high $\mathrm{fiO}_{2}$ did not lead to high levels of oxygen in brain tissue in most infants.

\section{NITRIC OXIDE DELIVERY WITH A NOVEL VENTILATOR CIRCUIT CONNECTOR - AN IN VITRO STUDY UNDER NEONATAL VENTILATORY CONDITIONS}

doi:10.1136/archdischild-2012-302724.1705

1J Mazela, ${ }^{1} \mathrm{~K}$ Chmura, ${ }^{2} \mathrm{C}$ Henderson, ${ }^{2} \mathrm{TJ}$ Gregory, ${ }^{3} \mathrm{M}$ Keszler, ' $\mathrm{J}$ Gadzinowski. ${ }^{1}$ Neonatology, Poznan University of Medical Sciences, Poznan, Poland; ${ }^{2}$ Research and Development, Discovery Laboratories, Inc.; ${ }^{3}$ Brown University, Women and Infants Hospital, Warrington, PA, USA

Introduction PPHN is treated with inhaled nitric oxide (iNO). A novel ventilator circuit connector (NVCC, AFECTAIR ${ }^{\circledR}$, Discovery Laboratories, Inc., Warrington, PA) has been developed to simplify the delivery of aerosols to patients receiving ventilatory support. We hypothesized that use of the NVCC for iNO delivery would substantially reduce NO consumption.

Aim To compare the NVCC with the SoC in the delivery of iNO under simulated neonatal ventilator conditions.

Material and Methods A pediatric/neonatal test system with Babylog ${ }^{\circledR}$ VN-500 with various inspiratory pressures, test lung, and ASL-5000 lung simulator were used. For SoC measurements, using a standard wye connector, the iNO was delivered per the manufacturer's instructions. With the NVCC, iNO was administered by introducing the NO via a tube attached directly to the NVCC. NO concentrations were measured with a $\mathrm{NOxBOX}^{\circledR}+$ analyzer and NO flow was recorded by Electronic Nitric Oxide flow controller abd titrated to $20 \mathrm{ppm}$ at the patient interface.

Results Compared with SoC, there was a 2 to 3 fold decrease in $\mathrm{NO}$ flow requirements to achieve desired iNO concentration with the NVCC. The delivery of $\mathrm{O}_{2}$ was not different between the study conditions. $\mathrm{NO}_{2}$ levels were slightly higher for the NVCC group, but never higher than $1.13 \mathrm{ppm}$.

Conclusion The NVCC significantly decreased the NO flow required for targeted delivery of $20 \mathrm{ppm}$. The NVCC allows for simplified therapeutic gas delivery with reduced $\mathrm{NO}$ utilization. These results warrant further study of NVCC on compatibility assessment with various modes of ventilation and delivery of other medical gases.

\section{VALUE OF ECHOCARDIOGRAPHY FOR THE DIAGNOSIS AND MONITORING OF EVOLUTION OF PERSISTENT PULMONARY HYPERTENSION IN NEWBORN}

doi:10.1136/archdischild-2012-302724.1706

'L Dimitiru, ${ }^{2}$ AG Dimitiru, ${ }^{3} \mathrm{M}$ Stamatin. ${ }^{1}$ Pediatric Cardiology, Medex Medical Center; ${ }^{2}$ Pediatric Cardiology; ${ }^{3}$ Neonatology, University of Medicine and Pharmacy, lasi, Romania

Purpose To evaluate the value of the echocardiographic exam for the diagnosis of the persistent pulmonary hypertension (PPHN) in the newborn infant.

Methods Patients, 41 newborns (aged 0-8 days) with PPHN induced by severe perinatal hypoxia, meconium aspiration syndrome, hyaline membrane disease, hypotermia, neonatal sepsis, infant of diabetic mothers, congenital cardiac malformations. Investigations of patients: clinical exam $\mathrm{PaO}_{2}$, ECG, chest X ray, Doppler echocardiography (ECHO). ECHO was repeated after 5-7 days in all patients.
Results Physical exam, cyanosis in the first 12 hours, tachypnea and/or a severe respiratory distress, systolic murmur on the left border of sternum. ECG: diastolic dysfunction of left ventricular (LV). Chest $\mathrm{X}$ ray: cardiomegaly (all cases). $\mathrm{PaO}_{2}$ : low values-all patients. $\mathrm{ECHO}$ aspects: enlargement of the right chambers; severe tricuspid regurgitation with the peak velocity $3-4 \mathrm{~m} / \mathrm{sec}$; mitral regurgitation (12/41 of cases), left-to-right shunt across foramen ovale and/or ductus arteriosus (30/41 of cases), enlargement of the pulmonary artery and severe pulmonary regurgitation, septal hypertrophy (11/41 of cases); impaired LV relaxation with normal systolic function; congenital heart diseases (7). Repeated ECHO revealed in most of the cases diminished or no right-to left shunt across ductus arteriosus or foramen ovalae correlate with clinical improvement and disappearance of cyanosis.

Conclusions Echocardiographic exam, beside clinical exam and history of the disease, is un important element for the diagnosis and follow up of evolution by the specific treatment applied for PPHN in the newborn with cyanosis and this investigation must be performed early after birth.

\section{RATE AND MANAGEMENT PATTERN OF CONGENITAL DIAPHRAGMATIC HERNIA AT MATERNITY HOSPITAL, KUWAIT}

doi:10.1136/archdischild-2012-302724.1707

MM Al Essa. Pediatrics, Faculty of Medicine, Kuwait University, Kuwait, Kuwait

Introduction \& Objectives: Congenital diaphragmatic hernia $(\mathrm{CDH})$ occurs in around 1 in 2000 live births and associated with high mortality rate reaching up to $50 \%$, even with prenatal diagnosis and recently advanced and extensive neonatal intensive care management. The objectives of this study are to document the incidence, clinical experience and outcomes of congenital diaphragmatic hernia $(\mathrm{CDH})$ in newborn infants admitted to the neonatal unit at Maternity Hospital, Kuwait.

Methods and Materials A retrospective chart review of all newborn infants admitted to the neonatal unit at Maternity Hospital in Kuwait from January 2007 till December 2010. Demographic data of the babies were collected along with variables involving the levels of sickness and therapeutic interventions.

Results A total of 21 cases of $\mathrm{CDH}$ were admitted to the unit over the four years period. The gestational age ranged from 33 to 40 weeks. 13 cases died giving a mortality rate of $61.9 \%$. All the cases received mechanical ventilation, and 16 of them was high frequency ventilation (HFO), while almost half of the cases received nitric oxide gas treatment. Almost all the cases were on $100 \%$ oxygen.

Conclusions Our results showed a little higher mortality than other centers worldwide, in spite our management pattern was not different. More cases need to be collected from the neonatal units in the other hospitals in Kuwait to compare the mortality rates and patterns of management.

\section{ROLE OF COMPUTED TOMOGRAPHY (CT) IN PEDIATRIC RESPIRATORY DISEASES}

doi:10.1136/archdischild-2012-302724.1708

HAA Bin-Nakhi, S Sadeq, N Alsayed, M Abdul Nabi. Pediatrics, Adan Hospital, Kuwait, Kuwait

Respiratory illness is one of the major causes of morbidity and mortality in children. CT lung has some advantages over plain chest radiography but exposes the child to more radiation. This study was carried to determine the value of performing lung CT in the management of children with respiratory diseases and to summarize the main indications for ordering pulmonary CT in Pediatrics. 
Subject \& Method A hospital based, retrospective case-review study was carried out in Pediatric department, Al-Adan Hospital, State Of Kuwait. The files of all patients less than 12 year age, and for whom CT lung was performed during the last five years (20002005) were reviewed.

Results Thirty patients for whom CT lung was performed were reviewed. The main indication for requesting chest CT was persistent abnormal CX-ray findings $(90 \%)$ in a patient with uncontrolled wheeze and/or recurrent pneumonia. Findings of chest CT was the same as CX-ray findings (80\%) and more specific +/- new diagnosis $(20 \%)$. Results of chest CT influenced medical treatment only in seven of the cases (23\%) and led to surgical intervention in two patients $(0.06 \%)$, (hernia, cystic adenomatoidmalformation).

Conclusion Our data demonstrate that the result of CT lung in children doesn't alter clinical decision and management of most of the cases. Therefore CT lung should be requested mainly for the high risk patients, preferably by a pulmonologist and after discussing the chest $\mathrm{x}$-ray findings with a radiologist.

\section{ESTIMATION OF NEONATAL ENDOTRACHEAL TUBE RESISTANCE BY WATER MANOMETER}

doi:10.1136/archdischild-2012-302724.1709

'P Mallya, ${ }^{2} \mathrm{P}$ Webb, ${ }^{1,3} \mathrm{~S}$ Gupta. 'Paediatrics and Neonatal Medicine, University Hospital of North Tees, Stockton; ${ }^{2}$ Medical Physics, James Cook University Hospital, Middlesbrough: 3University of Durham, Durham, UK

Background The loss of pressures at the distal end of ET tube can be affected by the size and diameter of the ET tube and the flow rates.

Aim To study in vitro the pressure drop using varying sizes of ET tubes commonly used in preterm infants at different lengths, flow rates using different gases.

Methods We used Portex tubes for this in-vitro study. A water manometer (scale $0-300 \mathrm{~mm}$ ) was used to measure the pressure drop across ET tube. We used two different lengths (7 and $14 \mathrm{cms})$; different sizes $(2.5 ; 3.0 ; 3.5$ and $4.0 \mathrm{~mm}$ ), different flow rates (4 to 11 litres/min) and two different gases (medical air and 100\% oxygen) to assess the resistance across the ET tube. SPSS version 17 $®$ was used for statistical analysis. Data presented as mean(SD).

Results

1. There was no difference in the pressure drop at lengths of $7 \mathrm{cms}$ and $14 \mathrm{cms}$ respectively using air [120.6 (66.12) vs.127.3 (68.74) $\mathrm{cms}_{2} \mathrm{O}$; $\left.\mathrm{p}=0.297\right]$; but with $100 \%$ Oxygen there was statistically significant increase at $14 \mathrm{~cm}$ compared to $7 \mathrm{~cm}$ length [146.73 (72.94) vs. $130.48\left(72.94 \mathrm{cms} \mathrm{H}_{2} \mathrm{O}\right.$; $\mathrm{p}=0.015]$ at similar flow rates.

2. At all flow rates there was no difference in pressure drop at different tube diameters using air or $100 \%$ oxygen

3. There was a statistically significant $(p<0.05)$ increase in pressure drop at flow rates increasing from 4 to 11 litres $/ \mathrm{min}$.

Abstract 1709 Table 1 Comparison of pressure drop with air and oxygen

\begin{tabular}{lll}
\hline Flow (I/min) & $\begin{array}{l}\text { Pressure drop with Air } \\
\mathbf{C m s H}_{\mathbf{2}} \mathbf{0}(\mathbf{M e a n} / \mathbf{S D})\end{array}$ & $\begin{array}{l}\text { Pressure drop with oxygen } \\
\mathbf{C m s H}_{\mathbf{2}} \mathbf{0} \text { (Mean/SD) }\end{array}$ \\
\hline 4 & $42.4 / 6.4$ & $55 / 26.3$ \\
5 & $58.1 / 8.3$ & $74.5 / 20.3$ \\
6 & $77.9 / 10.8$ & $97.7 / 16.0$ \\
7 & $101.6 / 13.5$ & $129 / 14.6$ \\
8 & $125.9 / 16$ & $162.1 / 18.9$ \\
9 & $156.7 / 20$ & $198 / 25.6$ \\
10 & $197.9 / 25.7$ & $236.2 / 33$ \\
11 & $227.7 / 59.3$ & $182.4 / 129$ \\
\hline
\end{tabular}

Conclusion The results from this study could be utilised to optimise ventilation settings to achieve the desired pressure delivery using air or oxygen at different flow rates and with varying ETtube sizes.

\section{SEROPREVALENCE OF CHLAMYDIA PNEUMONIAE AND MYCOPLASMA PNEUMONIAE IN CHILDREN WITH CHRONIC COUGH}

doi:10.1136/archdischild-2012-302724.1710

${ }^{1}$ S Asilsoy, ${ }^{2}$ E Bayram, ${ }^{3} \mathrm{D}$ Can. ${ }^{1}$ Pediatric Allergy, Başkent Universitesi, Adana; ${ }^{2} D o k u z$ Eylül University; ${ }^{3}$ Dr Behcet Uz Çocuk Hastanesi, Izmir, Turkey

Background Chronic cough is one of the most common symptoms in children. Postinfectious etiologies plays an important role in chronic cough in childhood. The pathogenesis of the postinfectious cough may be related persistant inflammation and the epithelial damage in the upper and lower airways, with or without transient airway hyperresponsiveness. We evaluated Mycoplasma pneumoniae and Chlamydia pneumoniae serology and treatment in children referred with chronic cough.

Methods This study enrolled 41 children between 6 and 14 years of age having cough which lasted than 4 weeks. They were evaluated according to American College of Chest Physicians guideline. Pulmonary function test and chest x-ray were performed to all patients. M. pneumoniae and C. pneumoniae serologies were analayzed by ELISA. They were reevaluated with 2 to 4 weeks intervals until cough disappeared.

Results The study included 41 children, 27 of whom were female (65.9\%). The mean age was $8.00 \pm 1.96$ year. M. pneumoniae IgM positivity was found in $17.07 \%(7 / 41)$ of patients, C. pneumoniae IgM positivity in $2.85 \%$ (1/35), M. pneumoniae IgM and/or IgG positivity in $41.46 \%(17 / 41)$, C. pneumoniae IgM and/or IgG positivity in $25.7 \%$ (9/35). Symptoms were not improved alone with clarithromycine treatment so inhaled/nasal steroids were added according to diagnosis.

Conclusion In children with chronic chough, aged 6 to 14 years old, M. pneumoniae and C. pneumoniae play important roles in the etiology. Clarithromycine alone may not be enough in the treatment of chronic cough due to these agents, so the treatment should be planned according to clinical findings.

\section{THE RELATIONSHIP BETWEEN THE FIRST EPISODE OF WHEEZING AND MATRIX METALLOPROTEINASES-9 AND -2 AND TIMP-1 LEVELS IN PRETERM INFANTS}

doi:10.1136/archdischild-2012-302724.1711

${ }^{1} \mathrm{RG}$ Sezer, ${ }^{1} \mathrm{~A}$ Bozaykut, ${ }^{2} \mathrm{~A}$ Tanju, ${ }^{2} \mathrm{G}$ Aydemir, ${ }^{1} \mathrm{LP}$ Seren, ${ }^{3} \mathrm{~S}$ Hira, ${ }^{3} \mathrm{O}$ Ozcan. ${ }^{1}$ Department of Pediatrics, Zeynep Kamil Maternity and Childrens' Disease Training and Research State Hospital; '2Department of Pediatrics; ${ }^{3}$ Department of Biochemistry, GATA Teaching Hospital, Istanbul, Turkey

Background Elevated concentrations of matrix metalloproteinases (MMP) have been associated with neonatal morbidity. There are no data on the serum levels of MMP-2, MMP-9, tissue inhibitors of matrix metalloproteinase (TIMP-1) from preterm infants recovering from these morbidities. We aimed to compare MMP-2, MMP-9, and TIMP-1 levels in preterm and term infants hospitalized with their first episode of wheezing.

Methods We prospectively evaluated 18 preterm infants with a history of chronic lung disease, respiratory distress syndrome and 14 age- and sex- matched term infants who were admitted for a first episode of wheezing. We quantified total serum concentrations of MMP-2, MMP-9, and TIMP-1 to assess whether these serum markers levels were associated with wheezing with a history of oxygen therapy during the neonatal period.

Results Upon hospitalization for the first episode of wheezing, MMP-2 and TIMP-1 levels were higher in preterm infants than in term infants. 